\title{
Attosecond Electron Spectroscopy Using a Novel Interferometric Pump-Probe Technique
}

\author{
J. Mauritsson, ${ }^{1}$ T. Remetter, ${ }^{1}$ M. Swoboda, ${ }^{1}$ K. Klünder, ${ }^{1}$ A. L'Huillier, ${ }^{1}$ K. J. Schafer, ${ }^{2}$ O. Ghafur, ${ }^{3}$ F. Kelkensberg, ${ }^{3}$ \\ W. Siu, ${ }^{3}$ P. Johnsson, ${ }^{3}$ M. J. J. Vrakking, ${ }^{3,4}$ I. Znakovskaya, ${ }^{5}$ T. Uphues, ${ }^{5}$ S. Zherebtsov, ${ }^{5}$ M. F. Kling, ${ }^{5}$ F. Lépine, ${ }^{6}$ \\ E. Benedetti, ${ }^{7}$ F. Ferrari, ${ }^{7}$ G. Sansone, ${ }^{7}$ and M. Nisoli ${ }^{7}$ \\ ${ }^{1}$ Department of Physics, Lund Institute of Technology, P. O. Box 118, SE-221 00 Lund, Sweden \\ ${ }^{2}$ Department of Physics and Astronomy, Louisiana State University, Baton Rouge, Louisiana 70803-4001, USA \\ ${ }^{3}$ FOM-Institute AMOLF, Science park 113, 1098 XG Amsterdam, The Netherlands \\ ${ }^{4}$ Max-Born-Institut für Nichtlineare Optik und Kurzzeitspektroskopie (MBI), Max-Born-Straße 2 A, 12489 Berlin, Germany \\ ${ }^{5} 4$ Max-Planck-Institut für Quantenoptik, Hans-Kopfermann-Strasse 1, D-85748 Garching, Germany \\ ${ }^{6}$ Université Lyon 1; CNRS; LASIM, UMR 5579, 43 bvd. du 11 novembre 1918, F-69622 Villeurbanne, France \\ ${ }^{7}$ Politecnico di Milano, Department of Physics Istituto di Fotonica e Nanotecnologie, CNR-IFN \\ Piazza L. da Vinci 32, 20133 Milano, Italy \\ (Received 24 March 2010; published 27 July 2010)
}

\begin{abstract}
We present an interferometric pump-probe technique for the characterization of attosecond electron wave packets (WPs) that uses a free WP as a reference to measure a bound WP. We demonstrate our method by exciting helium atoms using an attosecond pulse (AP) with a bandwidth centered near the ionization threshold, thus creating both a bound and a free WP simultaneously. After a variable delay, the bound WP is ionized by a few-cycle infrared laser precisely synchronized to the original AP. By measuring the delay-dependent photoelectron spectrum we obtain an interferogram that contains both quantum beats as well as multipath interference. Analysis of the interferogram allows us to determine the bound WP components with a spectral resolution much better than the inverse of the AP duration.
\end{abstract}

DOI: 10.1103/PhysRevLett.105.053001

PACS numbers: $32.80 . \mathrm{Rm}, 32.80 . \mathrm{Qk}, 42.65 . \mathrm{Ky}$

Attosecond science [1,2] promises to achieve temporal resolution comparable to the duration $(\tau)$ of the light pulses, e.g., of the order of 100 as [3,4] or even below [5]. An important issue, however, is whether this is possible only to the detriment of spectral resolution, thus considerably limiting the scientific interest of such light sources. When attosecond pulses (APs) interact with atoms or molecules, they create broad electron wave packets (WPs), partly in the continuum, but often including also a number of bound states excited by direct absorption [6] and/or by shake-up processes [7]. A spectral resolution given by the Fourier limit, i.e., of the order of $1 / \tau$, prevents any detailed analysis of such complex WPs.

Because of the low intensity of currently available APs, most of the techniques used to characterize attosecond electron WPs have used as a probe an infrared (IR) pulse which is synchronized to the extreme ultraviolet (XUV) AP. The required XUV-IR synchronization is inherent to the AP generation process and several methodologies, such as chronoscopy [7], streaking [8,9], stroboscopy [10], and interferometry [11] have been demonstrated. All of these techniques require that the attosecond and IR pulses overlap temporally. The IR field is therefore not only probing the electron WP after it has been created but it also perturbs the formation. In addition, the spectral resolution of these techniques is Fourier limited.

Several techniques could be considered to achieve a spectral resolution higher than the inverse of the pulse duration. Although trains of pulses, i.e., frequency combs, have the potential to achieve extremely high spectral reso- lution [12], pairs of pulses, as in traditional Ramsey spectroscopy, might be easier to implement for time-resolved measurements [13]. In this Letter we present a novel interferometric technique, using a single AP and a delayed IR pulse, that resolves both issues previously discussed: The spectral resolution is much better than the Fourier limit of the exciting pulse and the delayed probe pulse does not perturb the excitation process (see Fig. 1).

In our method, an AP is used to excite a bound WP in an atom or a molecule and a delayed IR pulse, locked in phase with the XUV pulse, is used to probe it. Coincident with the creation of the bound WP, we also create a continuum WP, which serves as a reference. After a variable delay, the bound WP is ionized by the IR pulse, and both continuum WPs, created directly by the XUV pulse, or by the two-step (XUV + IR) process, interfere. The analysis of the interferogram obtained when measuring the photoelectron spectrum as a function of delay allows us to determine the spectral components of the bound WP. This technique enables us to obtain a spectral resolution given by the inverse of the IR-XUV delay, which is typically a few tens of femtoseconds, i.e., more than a factor 100 better than the Fourier limit of the excitation pulse. We demonstrate the technique experimentally using an AP with 350 as duration and central energy of $24 \mathrm{eV}$ which excites a broad WP in helium, including bound (unknown) and continuum (reference) components. A few-cycle IR pulse probes the bound WP, and analyzing the electron spectra allows us to recover the composition of the WP. 

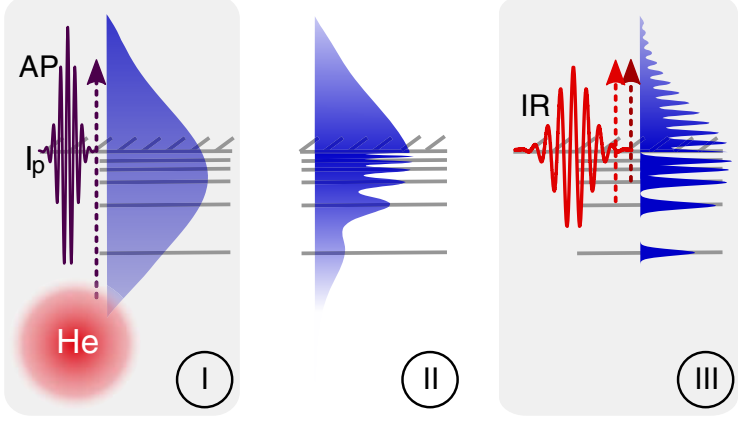

FIG. 1 (color online). Principle of attosecond electron interferometry. A broadband, AP with a spectrum centered on the ionization threshold of helium is used to coherently excite an electron WP consisting of a superposition of bound and continuum $p$ states (region I). The created WP evolves freely during a certain delay (II). The bound part of the WP is finally ionized by a few-cycle IR pulse (III), which is locked in phase to the AP and interferences with the previously created free WP are observed in the photoelectron spectra. In addition, several excited states can be excited leading to quantum beats in the ionization signal.

In the experiments, a linearly polarized phase stabilized 5-fs IR laser pulse is divided into a central and annular part using a mirror with a hole in the center. The polarization gating technique is used on the central part to obtain a laser pulse with a temporal window of linear polarization with duration of less than half an optical cycle [14]. This laser beam is focused into a xenon gas cell to generate XUV radiation via high order harmonic generation. The low order harmonics and the collinear IR radiation are removed using a $100 \mathrm{~nm}$ thick aluminum filter. This metallic filter also provides partial dispersion compensation of the intrinsic positive chirp of the emitted pulse [15], compressing it to a duration of 350 attoseconds. The APs are focused using a grazing incidence toroidal mirror into the active region of a velocity map imaging spectrometer (VMIS) [16], used to record the photoelectron momentum distributions.

The APs have a central frequency of $24 \mathrm{eV}$ with a bandwidth exceeding $10 \mathrm{eV}$ and excite helium from its ground state to a coherent superposition of bound and continuum $p$ states. At a controllable time delay the bound WP is ionized by the probe IR laser (bandwidth $0.53 \mathrm{eV}$ ), which is collinearly recombined with the AP using a second mirror with a hole in the center and which is focused by a spherical mirror. The IR intensity was close to $1 \times 10^{13} \mathrm{~W} / \mathrm{cm}^{2}$, well below that necessary to tunnel ionize $\mathrm{He}$ in its ground state, but high enough to induce "streaking" when both IR and XUV pulses overlap [3,17], allowing us to determine the delay between the two pulses. The XUV and IR beams are crossed with an effusive He gas jet emerging from a capillary incorporated into the repeller electrode of the VMIS [18]. Using a set of electrostatic lenses, the electrons emitted in the two-color photoionization process are accelerated onto multichannel plates coupled to a phosphor screen detector. The effusive gas jet allows us to obtain a gas density of $\sim 3 \times 10^{15} \mathrm{~cm}^{-3}$ in the interaction region, while keeping a low enough pressure close to the multichannel plates. Two-dimensional images are acquired with a CCD camera and used for the retrieval of the 3D initial velocity distribution [19].

A scan of the photoelectrons emitted in a small angle around the polarization axis in the upward direction is shown in Fig. 2. Both fields are vertically polarized. The color indicates the photoelectron intensity $S_{\text {exp }}(E, t)$ as a function of observation energy $E$ and delay $t$. When the two pulses overlap temporally (at 0 fs delay), the photoelectron spectra are streaked, indicating photoionization by an isolated AP in the presence of a laser field [3,17]. In the more interesting region where the AP precedes the IR probe, interference fringes are observed in the low-energy region of the spectrum, up to about $2 \mathrm{eV}$. As explained below, interferences fringes are expected also at higher energy, but the spectrometer resolution in this region prevents their observation [20]. This interference pattern is due to the multiple pathways leading to the same final continuum energy. During the delay, $t$, a continuum state with energy $E$ and a bound, stationary state with energy $E_{i}$ accumulate a phase difference $\left(E-E_{i}\right) t / \hbar$. The interference fringes, defined as the curves of constant phase difference, are therefore hyperboles, which become more closely spaced as the delay increases.

In Fig. 3, we present quantum calculations based on the single active electron approximation [6] under conditions that are close to those of the experiment. The probe has a 6 fs duration with a $\cos ^{2}$ shape and an intensity of $1 \times$ $10^{13} \mathrm{~W} / \mathrm{cm}^{2}$, while the AP duration is 180 as which is shorter that the 350 as in the experiment but chosen to match the experimental bandwidth [20]. The theoretical spectra as a function of delay, $S_{\text {theo }}(E, t)$, shows electrons emitted in the direction of the IR and XUV polarization axis. Interference fringes appear as soon as the excitation of the bound states is separated in time from their ionization by the probe field, meaning that we have two clearly delineated routes into the continuum. The hyperbolic shape

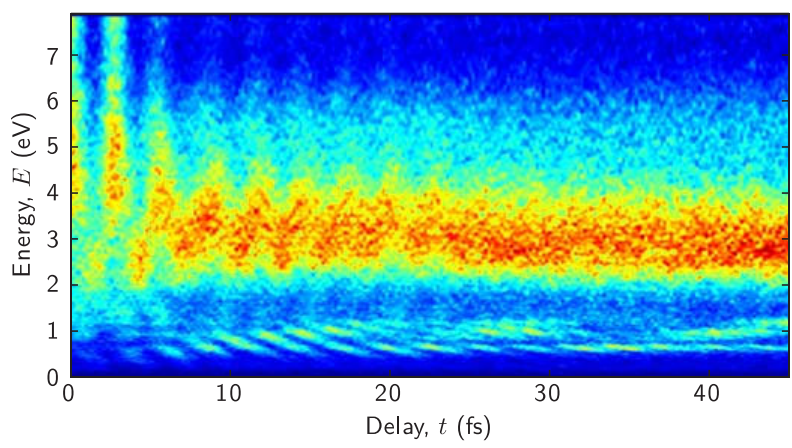

FIG. 2 (color online). Experimental photoelectron spectra in $\mathrm{He}$ as a function of delay. The electron distribution is recorded using a VMIS and the spectra are obtained by selecting a small collection angle along the polarization direction. The interference fringes are clearly visible at low energies. 
of the fringes is visible in the calculation and the fringe spacing in energy decreases as the delay increases.

A quantum beat signal $[21,22]$ is also visible in Fig. 3(a), due to simultaneous excitation of several bound states $(i, j)$. Interferences between quantum paths leading to the same final energy, for example $E_{i}+n \hbar \omega=E_{j}+n^{\prime} \hbar \omega^{\prime}, \omega, \omega^{\prime}$ being within the IR laser bandwidth and the number of photons $n$ and $n^{\prime}$ not necessarily the same (typically one or two), give rise to periodic structures in the ionization probability. The accumulated phase difference between the quantum paths, equal to $\left(E_{j}-E_{i}\right) t / \hbar$, is independent on the observation energy and the quantum beat signal appears as periodic vertical structures. The beat signal observed in Fig. 3 has a main periodicity of about 2 fs, corresponding to the beating between the outgoing electrons from the $2 p$ and the $3 p$ states. The periodicity of the beat signal carries information on the relative energy separation of the states involved, while the absolute timing of the beating depends on the relative phase of the pairs of states contributing to the signal. As we now discuss, analysis of the "direct-indirect" interferences, involving bound states and a reference continuum state allow us to go well beyond quantum beat spectroscopy.

To begin with, the different components of the excited WP can be extracted by Fourier analysis of the delaydependent photoelectron signal $S_{\text {theo }}(E, t)$. We first analyze the simulation shown in Fig. 3. The Fourier transform at all the possible observation energies yields a two dimensional function of the observation energy $E$ and the Fourier frequency (represented as an energy $E^{\prime}$ ). This function, $\mathcal{S}_{\text {theo }}\left(E, E^{\prime}\right)$, is presented as a color plot in Fig. 3(b) as a function of $E$ and $E^{\prime}$. Figure 3(c) shows a line out at $E=$ $4 \mathrm{eV}$. It exhibits six prominent peaks. The three lowest peaks are from the quantum beat between $3 p-4 p, 2 p-3 p$, and $2 p-4 p$ pairs of states, respectively, while the other peaks at 4.9, 5.6, and $7.4 \mathrm{eV}$ in Fig. 3(c) are due to the direct-indirect interferences involving the $4 p, 3 p$ and $2 p$ states, respectively. When the observation energy $E$ is varied, the Fourier frequency of the quantum beats does not change and they appear as vertical lines in Fig. 3(b). In contrast, the Fourier frequency $E^{\prime}$ of the direct-indirect interferences increases with $E$, since the accumulated phase difference between the direct and indirect ionization pathways is proportional to it. This linear relationship results in lines tilted at $45^{\circ}$ in Fig. 3(b). The energies of the bound intermediate states $(4 p, 3 p, 2 p)$ in the WP can be read directly from the intersections of the $45^{\circ}$ lines with the horizontal zero energy line. In addition, the relative strengths of the $45^{\circ}$ lines are directly related to the contributions from each bound state to the ionization signal.

Turning to the experimental data, we find that quantum beats and direct-indirect interferences are also present. Figure 4 presents an extended analysis of our experimental results. Figure 4(a) is a zoom of the low-energy region in Fig. 2, while Fig. 4(b) presents the Fourier transform of the experimental data. The observed $45^{\circ}$ lines which are characteristic for the direct-indirect interferences allow us to identify the composition of the bound WP and identify the contributions from the $3 p, 4 p$ and $5 p$ states. A weak indication of the $4 p-5 p$ quantum beat can also be seen as a beating with a 13 fs periodicity in Fig. 4(a) and as a vertical line near $0.5 \mathrm{eV}$ in Fig. 4(b). Additional information is encoded in the angular distributions since the two interference processes are fundamentally different and involve different angular momentum states. Figures 4(c)-4(f) present a more complete analysis making use of the full angular-resolved photoelectron distribution $F(E, \theta, t)$, which can be expanded as a sum of Legendre polynomials

$$
F(E, \theta, t)=\sum_{j=0}^{2 L_{\max }} \beta_{j}(E, t) P_{J}[\cos (\theta)],
$$

where $P_{J}$ is the Legendre polynomial of $J$ th order, $\beta_{J}$ an expansion coefficient, and $L_{\max }$ the maximum angular momentum component of the ionized wave function, which corresponds to the maximum absorbed angular momentum. After extraction of the individual expansion coefficients, $\beta_{J}(E, t)$, we apply the same Fourier analysis that was already applied to the photoelectron spectrum measured along the laser polarization axis. Figures $4(\mathrm{c})$ and $4(\mathrm{e})$ show the extracted expansion coefficients $\beta_{1}(E, t)$
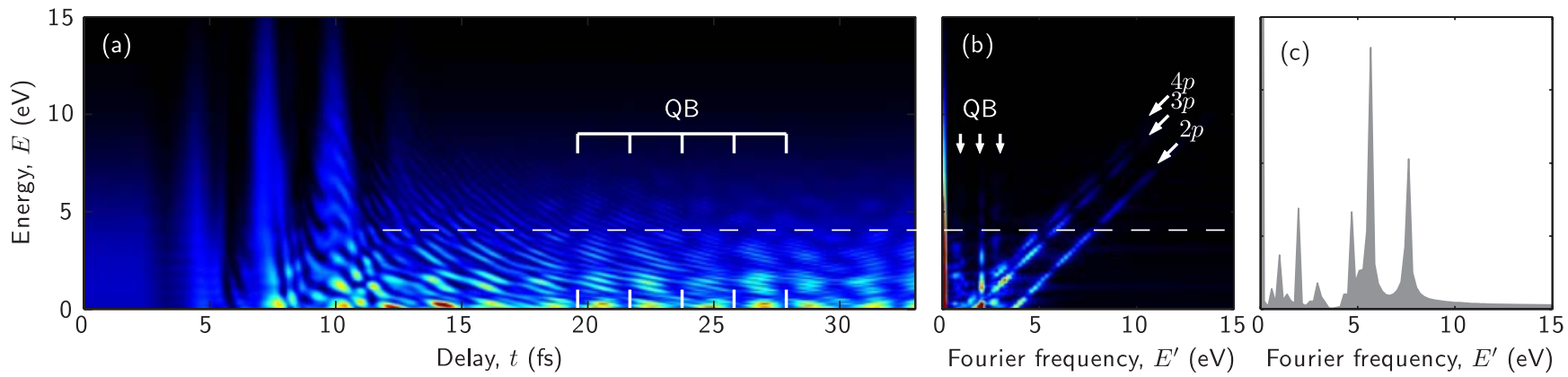

FIG. 3 (color online). (a) Calculated photoelectron spectra in He as a function of delay between the AP and the IR pulse. Interference fringes are clearly seen where the AP precedes the IR probe. (b) Fourier transform of the photoelectron spectrum allowing the identification of the states that form the bound WP. The beat signals from the $2 p, 3 p$ and $4 p$ states can be seen as vertical lines while the direct-indirect interference gives rise to contributions at an angle of $45^{\circ}$. A line out of the transform at $4 \mathrm{eV}$ is presented in (c). 

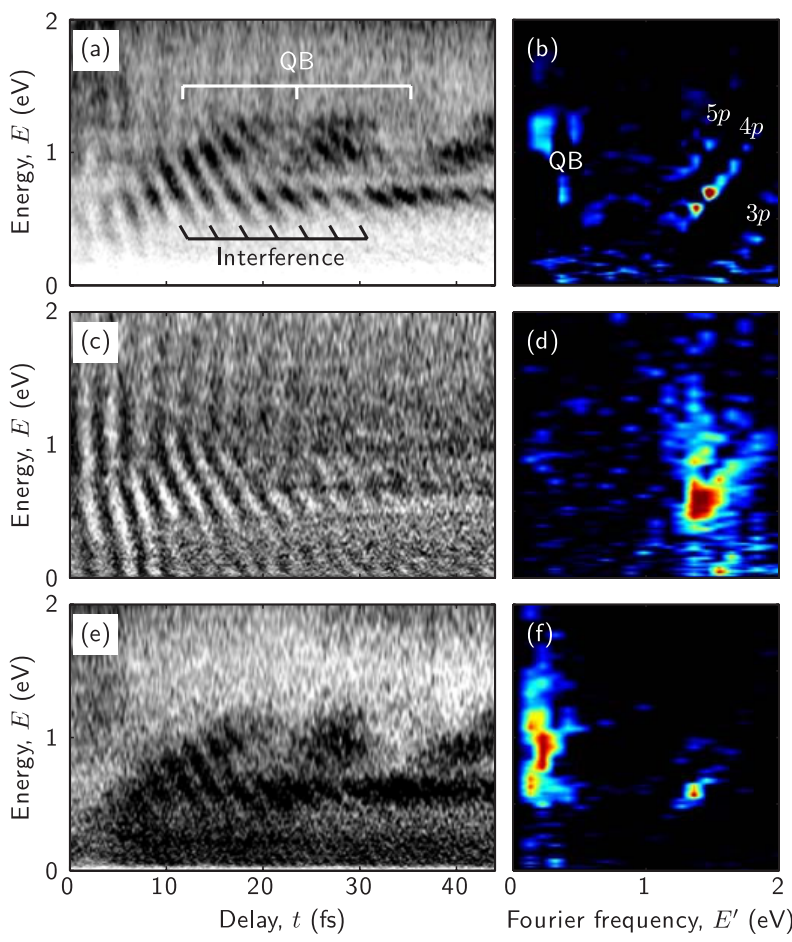

FIG. 4 (color online). Analysis of experimental results. (a) Zoom of the low-energy region in Fig. 2. (b) Fourier analysis showing the WP components $3 p, 4 p, 5 p$ as well as a quantum beat $4 p-5 p$. (c) $\beta_{1}(E, t)$ and (d) its Fourier analysis. (e) $\beta_{2}(E, t)$ and (f) its Fourier analysis.

and $\beta_{2}(E, t)$ respectively, together with their Fourier transforms in Figs. 4(d) and 4(f). Simple parity arguments dictate that interference between ionization processes that end in states of definite parity will appear in the even $J$ expansion coefficients, while interference between ionization processes that end in mixed parity states will appear in the odd $J$ expansion coefficients. The direct-indirect interference process involves electrons that have absorbed different numbers of photons resulting in a mixed parity state. Indeed, we see that the characteristic hyperbolic interference fringes appear clearly in $\beta_{1}(E, t)$ [Figs. 4(c) and 4(d)]. In contrast, the process that produces quantum beats results in states of definite parity. Accordingly, the quantum beat signal dominates $\beta_{2}(E, t)$ [Figs. 4(e) and 4(f)], thus confirming the interpretation presented above.

Using the above analysis, we can determine which states are excited by the $\operatorname{AP}(3 p, 4 p, 5 p)$ and obtain a measure of their relative strengths. Our ultimate goal is of course a complete characterization in both amplitude and phase. Determining the amplitudes and phases of a wavelike object by interference with a reference wave is a wellknown approach. The experiment and the analysis we have presented demonstrates that we can probe an attosecond electron WP using a coherent reference WP that is in the continuum. The method can be extended to include the retrieval of a phase associated with each WP component from the interferogram. This phase retrieval yields the phase difference between the two paths into the continuum and includes a phase contribution from both XUV and IR ionization steps. If it can be arranged that both ionization leading to the creation of the reference WP and ionization by the probe field do not add extra amplitude or phase variation or that these possible variations are well-known then a complete phase characterization can be made.

In conclusion we have demonstrated experimentally a new interferometric technique using a reference continuum WP and a delayed probe excitation. The method demonstrated can be used to probe the temporal evolution of bound or quasibound electron WPs, e.g., composed of autoionizing states [23] or created by shake-up [7] with high spectral and temporal resolution simultaneously, thus providing an increased precision when doing attosecond experiments.

This research was supported by the Marie Curie IEF (ATTOCO), RTN (XTRA), EST (MAXLAS) programs, the I3 Laserlab-Europe, the Swedish and European Research Councils, the National Science Foundation through grant No. PHY-0701372, the DFG through the Emmy-Noether program and the Cluster of Excellence: Munich Center for Advanced Photonics and the "Stichting voor Fundamenteel Onderzoek der Materie" (FOM), which is financially supported by the "Nederlandse Organisatie voor Wetenschappelijk Onderzoek" (NWO).

[1] P. M. Paul et al., Science 292, 1689 (2001).

[2] M. Hentschel et al., Nature (London) 414, 509 (2001).

[3] G. Sansone et al., Science 314, 443 (2006).

[4] E. Gustafsson et al., Opt. Lett. 32, 1353 (2007).

[5] E. Goulielmakis et al., Science 320, 1614 (2008).

[6] P. Johnsson et al., Phys. Rev. Lett. 99, 233001 (2007).

[7] M. Uiberacker et al., Nature (London) 446, 627 (2007).

[8] R. Kienberger et al., Science 297, 1144 (2002).

[9] J. Itatani et al., Phys. Rev. Lett. 88, 173903 (2002).

[10] J. Mauritsson et al., Phys. Rev. Lett. 100, 073003 (2008).

[11] T. Remetter et al., Nature Phys. 2, 323 (2006).

[12] M. Swoboda et al., Phys. Rev. Lett. 104, 103003 (2010).

[13] A. Pirri et al., Phys. Rev. A 78, 043410 (2008).

[14] I. J. Sola et al., Nature Phys. 2, 319 (2006).

[15] R. López-Martens et al., Phys. Rev. Lett. 94, 033001 (2005).

[16] A. T. J. B. Eppink and D. H. Parker, Rev. Sci. Instrum. 68, 3477 (1997).

[17] E. Goulielmakis et al., Science 305, 1267 (2004).

[18] O. Ghafur et al., Rev. Sci. Instrum. 80, 033110 (2009).

[19] M. J. J. Vrakking, Rev. Sci. Instrum. 72, 4084 (2001).

[20] The experimental spectrum was also a little modulated, leading to a minimum at $1.5 \mathrm{eV}$ and a maximum at $3 \mathrm{eV}$.

[21] J. A. Yeazell and C. R. Stroud, Jr., Phys. Rev. Lett. 60, 1494 (1988).

[22] A. ten Wolde et al., Phys. Rev. Lett. 61, 2099 (1988).

[23] L. Argenti and E. Lindroth, Phys. Rev. Lett. 105, 053002 (2010). 